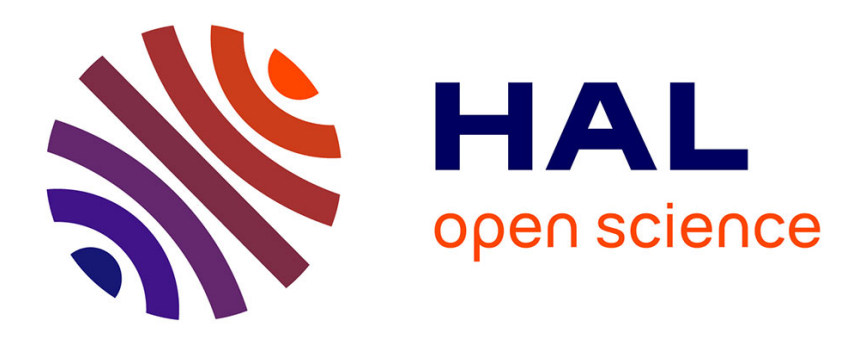

\title{
Sur la transformation de l'achromatisme optique des objectifs en achromatisme chimique
}

\author{
A. Cornu
}

\section{To cite this version:}

A. Cornu. Sur la transformation de l'achromatisme optique des objectifs en achromatisme chimique. J. Phys. Theor. Appl., 1874, 3 (1), pp.108-114. 10.1051/jphystap:018740030010801 . jpa-00236921

\section{HAL Id: jpa-00236921 https://hal.science/jpa-00236921}

Submitted on 1 Jan 1874

HAL is a multi-disciplinary open access archive for the deposit and dissemination of scientific research documents, whether they are published or not. The documents may come from teaching and research institutions in France or abroad, or from public or private research centers.
L'archive ouverte pluridisciplinaire HAL, est destinée au dépôt et à la diffusion de documents scientifiques de niveau recherche, publiés ou non, émanant des établissements d'enseignement et de recherche français ou étrangers, des laboratoires publics ou privés. 


\section{SUR LA TRANSFORMATION DE L'AGHROMATISME OPTIQOE DES OBJEGTIFS EN ACHROMATISME CHIMIQUE;}

Par M. A. CORNU.

(Note présentée au Congrès tenu à Lyon par l'Association française pour l'avancement des Sciences, séance du 22 août 1873 .)

Je m'étais proposé le problème suivant à l'occasion des travaux préparatoires de la Commission du passage de Vénus :

Transformer l'objectif d'une lunette astronomique, achromatisée pour les rayons visibles, de manière à obtenir, au foyer principul. d.' ipreuves photographiques dont la netteté soit comparable à celle des imagres optiques directes.

Des études antérieures sur la photographie du spectre ultraviolet $\left({ }^{1}\right)$ m'avaient conduit à examiner la répartition des foyers des diverses raies du spectre dans une lunette de spectroscope, formée avec un objectif achromatique. Une graduation en millimètres,

(') Foir le Compte rendu de l'Association française, Congrès de Bordeaux, t. I, p. 300 . 
tracée sur le tube de tirage d'une semblable lunette, permet d'observer les faits suivants : si l'on note successivement le tirage de la lunette, après avoir mis au point avec beaucoup de soin l'image des principales raies du spectre projetées sur le réticule, on remarquera que la distance focale diminue d'abord depuis la raie $\mathbf{A}$ jusqu'aux raies $\mathrm{B}, \mathrm{C}, \mathrm{D}$; il y a un minimum de distance focale vers la raic $b$, puis augmentation à partir de cette région; la raie $F$ forme son foyer à peu près à la même distance que $\mathrm{D}$, l'augmentation devient rapide à partir de $G$, si bien que le fover des raies de l'extrémité visible du violet se forme en arrière du foyer de l'extrémité rouge. La photographie permet d'aller beaucoup plus loin, et l'on peut suivre la marche très-rapidement croissante des distances focales des radiations ultra-violettes.

De ces faits résulte la remarque suivante : la répartition des foyers de la région violette dans un objectif achromatisé est précisément inverse de celle qu'aurait produite un objectif non achromatisé; en eflet, les rayons violets, étant les plus réfrangibles, ont une distance focale plus courte que les rayons rouges, lorsqu'on les fait réfracter à travers une lentille convergente de crown-glass : par exemple, l'addition d'une lentille divergente de flint-glass, qui tend à ramener les foyers de toutes les radiations dans un mème plan, produit donc une action plus énergique sur les rayons violets que sur les rayons rouges, puisqu'elle allonge le foyer des premiers d'une plus grande quantité que celui des derniers. 11 en résulte que, si par un moyen ou par un autre on diminue l'influence de la lentille divergente, on modifie la répartition des foyers des diverses radiations, telle qu'elle a été décrite plus haut, dans un sens qu'on peut aisément prévoir. Le foyer des rayons violets étant relativement moins repoussé que celui des rayons rouges (relativement au cas ordinaire), les rayons dont la distance focale est minimum se rapprochent du côté violet; l'achromatisme optique est alors altéré : en continuant à diminuer l'influence du verre diversent, on peut parrenir a placer la distance focale minimum dans la region comprine entre $\mathrm{G}$ et $\mathrm{H}$. On obtient alors l'achromatisme chimique; car cette région est, pour les radiations chimiques, l'analogue de la région du spectre visible comprise entre D et F, par l'énergie des impressions qu'elle produit sur les substances photographiques, autrement dit, le centre de gravité des radiations actiniques est situé 
vers le milieu de l'intervalle $\mathrm{G}$ et $\mathrm{H}$; comme dans le spectre visible ce point tombe aux environs de la raie $b$.

Quant au moyen de diminuer l'influence du verre divergent de flint-glass, le plus facile consiste simplement à l'écarter du verre convergent de crown-glass. On se rend compte aisément de cet effet en poussant la condition à l'extrème; en effetet, si l'on plaçait le verre livergent au foyer mème du verre convergent, son influcnce serait nulle; c'est en le rapprochant que son action augmente, jusqu'au moment où les deux verres sont en contact. Évidemment, dans une position intermédiaire, l'effet du flint-glass sera intermédiaire : on est donc assuré, par un écartement convenable des verres, d'arriver à l'achromatisme chimique; car on peut parcourir tous les degrés de répartition relative des foyers, depuis l'achromatisme optique qu'on peut considérer comme un achromatisme chimique dépassé, jusqu'au non-achromatisme $\left({ }^{1}\right)$.

(') J'ai vérifıé expérimentalement toutes ces déductions à l'aide d'un appareil assez simple: on dispose un objectif dont les verres peuvent ètre écartés à des distances variables (jusqu'à 2 ou 3 pour $ı 0$ de la distance focale principale); en avant de cet objectif, on place un prisme dont l'angle est de 8 à 12 degrés, suivant la réfrangibilité de la matière, et dont l'une des faces est argentée, de manière à fonctionner à la fois comme prisme d'angle double et de miroir renvoyant les rayons dans la même direction. En arrière de l'objectif, on place un oculaire positif et un réticule portés par un tirage avec graduation : l'axe de cet oculaire est légèrement incliné sur l'axe principal de l'object $\backslash$ l, de manière à laisser place à une fente éclairée par la lumière du soleil ou d'une étincelle d'induction. Il est facile maintenant de se rendre comple du fonctionnement de l'appareil. La fente étant disposée verticalement au-dessous de l'oculaire, dins le plan focal principal de l'objectif, les rayons émanés de cette fente sont wulu parallèles après leur passage à travers l'objectif, réfractés par la première face du prinme, riflechis par la seconde, réfractés une seconde fois (dans le sens de 1.1 luplication de la dispersion), puis ramenés sur l'objectif : le prisme n'altérant nullement le parallelisme des rayons si ses faces sont bien planes, les rayons vont con verger dans le plan focal principal en un point situé au-dessus de la fente : c'est là qu'on place le réticule, entrainé par le mouvement commun du tube de tirage et de l'oculaire. En donnant au prisme un mouvement de rotation autour de son arête, on peut amener chaque raie sous le fil vertical du réticule et la mettre exactement au point sur ce $\mathrm{fil}$; on peut donc ainsi mesurer la position relative des fovers de toutes les raies. On repète la mim. sır ie d'observations pour les distances convenablement choisies des deux verres. 't l'on verifie les modifications successives d'achromatisme

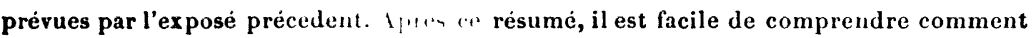
ces expériences constituent une villuble méthode optique pour l'étude rigoureuse de l'achromatisme.

II n'est pas inutile d'ajouter que l'écartement des verres produit une diminution notable de la distance focale principale; arec les verres usités d'ordinaire. la variation de distance focale est sept ou huit fois plus grande que la variation de la distance des verres. 
On peut donner une démonstration tout à fait élémentaire de cette méthode d'achromatisme chimique par écartement des verres, en supposant les lentilles sans épaisseur.

Soient $p$ et $p^{\prime}$ les distances de deux foyers conjugués d'une lentille convergente formée d'un verre dont l'indice soit $n$ pour un rayon donné; on a la formule bien connue

$$
\frac{\mathbf{I}}{p}+\frac{\mathbf{I}}{p^{\prime}}=(n-\mathbf{I})\left(\frac{\mathrm{I}}{\mathrm{R}}+\frac{\mathbf{I}}{\mathrm{R}^{\prime}}\right)=(n-\mathrm{I}) \mathbf{P},
$$

en désignant, pour abréger, la somme des courbures par $\mathrm{P}$.

$\mathrm{La}$ formule analogue pour une lentille divergente sera

$$
\frac{1}{q}-\frac{1}{q^{\prime}}=\left(n^{\prime}-1\right) Q .
$$

Si ces deux lentilles sont accouplées au contact (comme dans les objectifs ordinaires), on calculera la distance focale principale $\varphi$ du système, en faisant $p=\infty, q=p^{\prime}, q^{\prime}=\varphi$.

Si les deux verres sont écartés à la distance $a$, il faudra substituer $p=\infty, p^{\prime}+a=q, q=\varphi$; et, en remplaçant la lettre $p^{\prime}$ par $f$, pour rappeler que $p^{\prime}$ est la distance focale principale du verre convergent, on aura

$$
\frac{\mathrm{I}}{f-a}-\frac{\mathrm{I}}{\varphi^{\prime}}=\left(n^{\prime}-1\right) Q
$$

avec

$$
\frac{1}{f}=(n-1) \mathbf{P} .
$$

L'écartement des verres doit rester dans des limites très-étroites ( I à I $\frac{1}{2}$ pour I oo de la distance focale totale, environ o, o $2 f$ à $0,03 f$ ).

On peut écrire ainsi le terme

$$
\frac{\mathrm{I}}{f-a}=\frac{\mathrm{I}}{f\left(1-\frac{a}{f}\right)}=\frac{1}{f}\left(1-\frac{a}{f}\right)^{-1} .
$$

La formule (3) devient, après substitution de la valeur (4) de $f$,

$$
\frac{1}{\varphi^{\prime}}=\left(1-\frac{a}{f}\right)^{-1} \mathbf{P}(n-1)-Q\left(n^{\prime}-1\right) \cdot
$$

Pour interpréter cette équation d'une manière toute géométrique, 
nous aurons recours à la considération d'une courbe qui joue un grand ròle dans la représentation graphique des conditions d'achromatisme, et que pour cette raison j'appellerai courbe d'achromatisme.

On l'obtient en construisant les points dont l'abscisse $x=n-\mathbf{I}$ et l'ordmuce $y=n^{\prime}-1$ ont respectivement pour valeurs les indices diminués de l'unité des deux verres, indices correspondant à la mème couleur, ou mieux à la mème raie.

Ainsi, les points $\mathrm{A}, b, \mathrm{G}, \mathrm{H}$ de la courbe ci-jointe ( $f \mathrm{fg}_{\mathrm{g}} \mathrm{I}$ ) ont été formén c.ll prronant pour abscisses les indices, moins l'unité,

Fig. I.

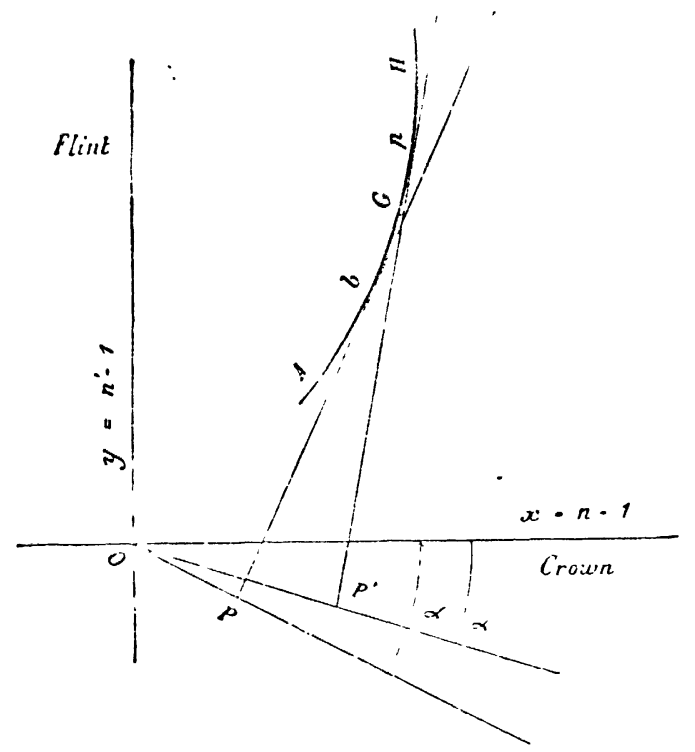

du crown-glass correspondant aux raies $A, b, \mathrm{G}, \mathrm{H}$, et pour ordonnées les indices moins l'unité du flint-glass correspondant aux mimer raire. La substitulion dé valeurs numériques montre ' $j$ u arce ces genres de verres la courbe tourne sa convexité vers l'axe (ies $x$ (crown-glass), et que la direction moyenne de l'arc de courbe passe au-dessoun de l'nifine at qu clle fait un angle d'environ 60 degrés ance l'an lies $x$.

La condition d'achromatisme, représentéc par l'équation (5), est 
que, pour toutes les valeurs correspondantes de $x$ et de, , la variariation de $\varphi$ soit aussi petite que possible. Substituant

il vient

$$
x=n-1, \quad y=n^{\prime}-\mathbf{1},
$$

$$
\frac{\mathrm{I}}{\varphi^{\prime}}=\left(1-\frac{a}{f}\right)^{-1} \mathbf{P} x-Q y ;
$$

expression de la forme

$$
x \cos \alpha-y \sin \alpha,
$$

ce qui conduit, par identification, à

$$
\frac{\cos \alpha}{\left(1-\frac{a}{f}\right)^{-1} \mathbf{P}}=\frac{\sin \alpha}{Q} \quad \text { ou } \quad \operatorname{tang} \alpha=\frac{Q}{\mathbf{P}}\left(1-\frac{a}{f}\right) \text {. }
$$

On conclut, de cette identification, que l'inverse de la distance focale résultante $\varphi^{\prime}$ est proportionnelle à la projection du rayon vecteur de la courbe d'achromatisme, sur une droite faisant avec l'axe des $x$ un angle égal à $-\propto$. Géométriquement, la condition d'achromatisme devient celle-ci : déterminer la direction telle que la projection de l'arc de courbe, considérée dans toute l'étendue qui correspond aux indices des rayons utiles, soit minimum.

Il est évident que cette condition sera remplie, si l'on projette suivant la droite qui joint les deux extrémités de la courbe, ou mème si l'on projette l'arc suivant la tangente au point correspondant à la raie $b$, qui, dans les rayons visibles, représente ce que symboliquement j'ai appelé le centre de gravité du spectre visible.

Pour l'achromatisme des rayons chimiques, la direction de la projetante est celle de la tangente en un point $\mu$ situé vers le milicu de l'intervalle GH. Comme la courbe est convexe, cette tangente fait un angle plus grand que le précédent avec l'axe des $x$ : donc $\alpha$ doit diminuer.

L'équation (6) montre qu'en effet, pour diminuer $\alpha$, il faut augmenter $a$. Donc l'écartement des verres transforme bien l'achromatisme des rayons visibles en achromatisme chimique. La règle expérimentale définie plus haut est donc démontrée.

Le raccourcissement correspondant du foyer est également évident; car la distance $\mathrm{OP}^{\prime}$, inverse de $q^{\prime}$, est plus grande que OP. 
En résumé, étant donnée une lunette astronomique achromatique dont l'objectif est formé d'une lentille convergente de crownglass et d'une lentille divergente de flint-glass, on la transformera en un objertif susceptible de donner des images photographiques d'une netteté aussi satisfaisante que possible, en écartant les deux lentilles d'une quantité dépendant de la nature de ces deux verres. Avec les verres unitís 'no optique, un écartement de I $\frac{1}{2}$ pour roo de la distance focale de l'objectif est suffisant, et le foyer chimique est très-roisin du foyer optique.

Quant aux aberrations produites par cet écartement des verres, je me suis assuré, par expérience, qu'ils sont entièrement négligeables. J'ai étudié à ce point de vue plusieurs lunettes de différents foyers, en particulier une excellente lunette de soo millimètres d'ouverture et de $\mathbf{I}^{\mathrm{T}}, 40$ de distance focale. Avec un écartement de 15 millimètres des deux verres, j’ai réussi à photographier une échelle divisée en millimètres, placée à 40 mètres de distance; les traits étaient parfaitement distincts; le rapport des pleins aux vides était bien reconnaissable et l'on pouvait au microscope mesurer avec un micromètre la trentième partie des intervalles. Un relevé micrométrique d'une épreuve très-soignée fait sur une plaque d'argent, d'après le procédé de Daguerre, m'a permis de constater que, sur toute l'étendue de l'échelle qui comprenait près de six cents traits et qui sous-tendait un angle de 45 minutes, il n'y avait aucune déformation systématique.

En résumé, la théorie et l'expérience montrent qu'un objectif bien achromatisé pour les rayons visibles peut être rendu achromatique au mime degré pour les rayons chimiques, par un simple écartement des deux verres.

L'écartement le plus convenable doit être cherché par une série d'essais méthodiques : il ne dépasse pas en général $1 \frac{1}{2}$ pour 1 oo de la distance focale principale primitive de l'objectif. On atteint aisément l'écartement qui correspond à une distance focale jouissant de la propricté précicuse de domner a la fois une image visible passable et une image photographique parfaite.

En terminant, je dirai que la méthode de transformation d'achromatisme est générale, et qu'elle peut s'appliquer à la solution de bien de's fuestions de physique, d'astronomie et de micrographie. 